\title{
Ion-Specific and pH-Dependent Hydration of Mica-Electrolyte Interfaces
}

\author{
Simone R. van Lin, ${ }^{\dagger}$ Kara K. Grotz, ${ }^{\ddagger}$ Igor Siretanu, ${ }^{\dagger}$ Nadine Schwierz, ${ }^{\ddagger}$ and Frieder Mugele $*^{\dagger}$ \\ ${ }^{\dagger}$ Physics of Complex Fluids Group and MESA+ Institute, Faculty of Science and Technology, University of Twente, P.O. Box 217, \\ 7500 AE Enschede, The Netherlands \\ ${ }^{\ddagger}$ Department of Theoretical Biophysics, Max Planck Institute of Biophysics, Max-von-Laue-Straße 3, 60438 Frankfurt (Main), \\ Germany
}

Supporting Information

ABSTRACT: Hydration forces play a crucial role in a wide range of phenomena in physics, chemistry, and biology. Here, we study the hydration of mica surfaces in contact with various alkali chloride solutions over a wide range of concentrations and $\mathrm{pH}$ values. Using atomic force microscopy and molecular dynamics simulations, we demonstrate that hydration forces consist of a superposition of a monotonically decaying and an oscillatory part, each with a unique dependence on the specific type of cation. The monotonic hydration force gradually decreases in strength with decreasing bulk hydration energy, leading to a transition from an overall repulsive $\left(\mathrm{Li}^{+}, \mathrm{Na}^{+}\right)$to an attractive $\left(\mathrm{Rb}^{+}, \mathrm{Cs}^{+}\right)$ force. The oscillatory part, in contrast, displays a binary character, being hardly affected by the presence of strongly hydrated cations $\left(\mathrm{Li}^{+}, \mathrm{Na}^{+}\right)$, but it becomes completely

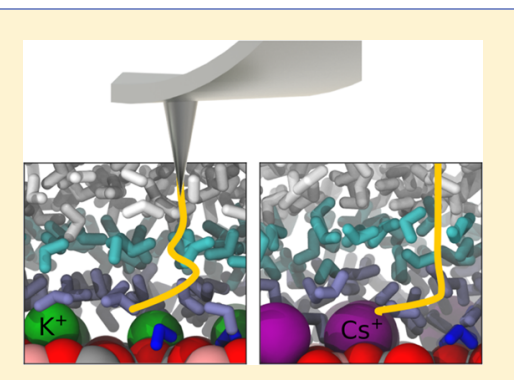
suppressed in the presence of weakly hydrated cations $\left(\mathrm{Rb}^{+}, \mathrm{Cs}^{+}\right)$, in agreement with a less pronounced water structure in simulations. For both aspects, $\mathrm{K}^{+}$plays an intermediate role, and decreasing $\mathrm{pH}$ follows the trend of increasing $\mathrm{Rb}^{+}$and $\mathrm{Cs}^{+}$ concentrations.

$\mathrm{H}$ ydration forces are essential for the stability of many colloidal systems, ${ }^{1}$ the assembly of soft biological and nonbiological matter on molecular and supramolecular scales, ${ }^{2-4}$ the swelling of clays, ${ }^{5}$ wetting, ${ }^{6,7}$ adhesion, ${ }^{8}$ and lubrication, ${ }^{9}$ as well as the efficiency of catalysts. ${ }^{10}$ In all these cases, "reactants" have to find each other under the influence of molecular interaction forces in the presence of an ambient solvent that frequently contains other solutes including, in particular, salts. ${ }^{11}$ At separations of a few nanometers, these interaction forces and their salt dependence are reasonably well described by the classical Derjaguin, Landau, ${ }^{12}$ Verwey, and Overbeek $^{13}$ theory of colloid science in combination with charge regulation equilibria. ${ }^{14,15}$ "The last nanometer", however, is generally governed by short-range and hydration forces. On this scale, at the boundary between physics and chemistry, the origin of the forces and their dependence on the water composition (salt content, specific ion effects, $\mathrm{pH}$ ) are much more difficult to characterize, and the contributions of different aspects such as surface hydration, bulk ion hydration, and direct ion-substrate interaction are much more difficult to disentangle. $^{16}$

In the present work, we focus on the effect of ions on hydration forces at atomically smooth mica-electrolyte interfaces, a well-characterized system that has been studied intensively since the pioneering works using the surface forces apparatus (SFA) by Israelachvili and Pashley ${ }^{17,18}$ in the 1980s. Recent advances in experimental techniques such as X-ray reflectometry and surface diffraction, ${ }^{19-21}$ nonlinear optical spectroscopies, ${ }^{22}$ and atomic force microscopy $(\mathrm{AFM})^{23-25}$ along with powerful molecular dynamics (MD), ${ }^{26,27}$ Monte Carlo, $^{28}$ and density functional theory ${ }^{29}$ simulations have produced detailed complementary insights into the structure and dynamics of interfacial water and cast doubt on some of the interpretations of earlier SFA experiments. However, a consistent picture is yet to emerge. For instance, hydration forces on mica have been reported to display either a monotonic exponential decay ${ }^{30}$ as observed for silica ${ }^{31,32}$ and many biological systems ${ }^{33,34}$ or an oscillatory character. ${ }^{18}$ It has been argued that hydration forces, in particular oscillatory ones, require the presence of ions. ${ }^{17,18}$ Consequently, specific properties of the ions such as their hydration in bulk solution and/or ion-ion correlations would determine the characteristic spacing of force oscillations. ${ }^{25,35-37} \mathrm{X}$-ray reflectivity and molecular simulations, however, typically report structural oscillations determined by the size of water molecules. ${ }^{20,26,27}$ Optical sum frequency generation measurements, on the other hand, report substantial orientational order of water molecules due to the alignment of water dipoles by local electric fields at polar surfaces. $^{38,39}$

Compared to classical force-distance measurements with the SFA, ${ }^{18,30,35,37} \mathrm{AFM}^{23,24}$ provides several advantages. In particular, it avoids confinement effects over a laterally extended contact area and thereby guarantees much better that ions and water molecules have sufficient time to

Received: February 21, 2019

Revised: April 5, 2019

Published: April 11, 2019 
equilibrate their configuration during the measurement. Moreover, much more local information can be extracted, yielding three-dimensional tip-sample force fields with atomic resolution and forces approaching the ones experienced by individual molecules, as suggested by the agreement between experiments and molecular simulations. ${ }^{40-44}$ However, the complexity and measurement time required to record such three-dimensional force maps have so far hampered systematic AFM investigations for a substantial range of ionic species and concentrations, leaving at present a somewhat scattered picture of isolated observations.

In the present work, we harvest the speed and robustness of one-dimensional high-resolution AFM force mapping to provide the first comprehensive overview of hydration forces and structures at mica-water interfaces in the presence of alkali chloride salts over a wide range of concentrations and $\mathrm{pH}$ values. Combining AFM spectroscopy and MD simulations, we demonstrate that the appearance of structured layers of water is indeed an intrinsic property of the pure system in the absence of any added salt. For all fluid compositions, the hydration forces can be described by a superposition of an oscillatory and a monotonically decaying part. Both respond in a different manner to the presence of salt and variation of $\mathrm{pH}$. While periodicity and range are largely independent of salt concentration and $\mathrm{pH}$, the monotonic repulsion disappears with increasing cation size. Most strikingly, the oscillatory part disappears for weakly hydrated $\mathrm{Rb}^{+}$and $\mathrm{Cs}^{+}$ions beyond a certain threshold concentration. Molecular dynamics simulations demonstrate how the latter arise from the disruption of the intrinsic mica hydration layers by adsorbed bulky cations.

\section{METHODS}

Amplitude Modulation (AM)-AFM Spectroscopy. Dynamic force spectroscopy measurements were performed using commercial Asylum Research Cypher ES equipped with photothermal excitation. The experiments were performed in liquid in a closed cell that allows exchange of liquid and control of the temperature (which was kept around $28{ }^{\circ} \mathrm{C}$ ). The cantilever was immersed in a droplet of liquid $(0.2 \mathrm{~mL})$ that was sandwiched between the substrate and the top of the cell. Fluid was exchanged using glass syringes connected to the tubing. The drop volume was replaced at least 25 times by the new fluid and was left to equilibrate for $30 \mathrm{~min}$ before starting the measurements. Force spectroscopy was performed in the amplitude modulation (AM) mode. ${ }^{45-47}$ During this procedure, the amplitude and phase of the cantilever deflection were tracked as a function of $\mathrm{z}$ piezo distance. For the measurement of the tip-sample interactions, the cantilever was driven at a fixed frequency $\left(\omega \approx 0.97 \omega_{0}\right.$, where $\omega_{0}$ $\approx 450 \mathrm{kHz}$ ) by an intensity-modulated blue laser diode that was focused on the gold-coated topside of the cantilever close to its base. AFM measurements were performed with an oscillation amplitude of $A=0.2-0.4 \mathrm{~nm}$. For each amplitude- and phase-distance curve, the cantilever was ramped over $5 \mathrm{~nm}$. For each fluid composition, we typically measured 200 approach curves. A threshold value was used to limit the approach of the tip and sample. This set point was set such that a linear part in the deflection signal was observed due to the tip contacting the surface. The recorded curves were aligned on the first oscillation found in the force gradient profile and were set at 0.25 $\mathrm{nm}$, where the maximum of the first oscillation is found. This sets the 0 , where we find the linear region in the deflection data indicating the tip-substrate contact.

Experimental Preparation. The mica substrate was glued using epoxy to a steel puck, which is magnetically clamped to the piezo stage of the AFM. The muscovite was cleaved with adhesive tape before each experiment. Each experiment started by flushing the system with purified water. The electrolyte solutions were prepared by dissolving the salts $(\mathrm{NaCl}, \mathrm{KCl}, \mathrm{LiCl}, \mathrm{RbCl}$, and $\mathrm{CsCl} 99 \%$ purity) in purified water. The $\mathrm{pH}$ is controlled by adding either $\mathrm{NaOH}$ or $\mathrm{HCl}$ to the solution. The $\mathrm{pH}$ of the electrolyte solutions without adjustment is around 6. All chemicals used were purchased from Sigma-Aldrich.

\section{CANTILEVERS}

Silicon ultrahigh-frequency cantilevers (ARROW-UHFAuD, nanoworld) with gold coating (detector side) were used. The length and width were 35 and $42 \mu \mathrm{m}$, respectively. The tip has an arrow shape, and the force constant $(k)$, resonant frequency $(f)$, and quality factor $(Q)$ of the first eigenmode as determined in the liquid were in the range $k=1.23-3.35$ $\mathrm{N} / \mathrm{m}, f=600-1000 \mathrm{kHz}$, and $Q=4-6$, respectively. The above values have been determined in purified water (Millipore, resistivity $18.2 \mathrm{M} \Omega \mathrm{cm}$ ). The force constant was determined by using the thermal method. Prior to use, the cantilevers were cleaned by putting them in a bath of $1: 1$ ethanol/isopropanol for $15 \mathrm{~min}$, after which they were dried using air and placed in a plasma cleaner (PDC-32G-2, Harrick Plasma) for $20 \mathrm{~min}$. The tip radius was determined after data collection from scanning electron microscopy imaging and was found to be around $5 \mathrm{~nm}$ for all used levers in this study (which is an upper limit since this was measured after tip calibration where the tip is pressed into contact). Relatively stiff cantilevers were used to prevent mechanical instabilities in the presence of attractive force gradients.

MD Simulations. We simulated muscovite mica $\mathrm{KAl}_{3} \mathrm{Si}_{3} \mathrm{O}_{10}(\mathrm{OH})_{2}{ }^{48}$ of dimensions $3.5 \times 5 \times 4.5 \mathrm{~nm}^{3}(5$ layers with 48 unit cells each) using the CLAYFF force field ${ }^{49}$ in simulation boxes of dimensions $3.5 \times 5 \times 11 \mathrm{~nm}^{3}$, where the (001) plane was set to be orthogonal to the $z$-axis of the simulation box. The exposed plane naturally contains potassium ions that were removed; the remaining mica crystal was hence negatively charged. We solvated the mica in TIP3P water. ${ }^{50} \mathrm{We}$ added $101 \mathrm{Li}^{+}, \mathrm{Na}^{+}, \mathrm{K}^{+}$, or $\mathrm{Cs}^{+}$ions and for each system $5 \mathrm{Cl}^{-}$ions to neutralize and to adjust to the experimental salt concentrations of $50 \mathrm{mM} \mathrm{LiCl}, \mathrm{NaCl}, \mathrm{KCl}$, or $\mathrm{CsCl}$, respectively. We chose a recently developed ion force field that is designed to reproduce both thermodynamic and kinetic properties of aqueous solutions. ${ }^{51}$ To mimic the measurements in pure water, protons were introduced (see Supporting Information for details) to neutralize the system. Inserting the ions directly in the solution leads to a random adsorption of cations at the mica surface during the equilibration phase (Supporting Information Figure 11).

MD simulations were performed with GROMACS (versions 5.1.3 and 2016.4)..$^{52}$ Energy minimization was followed by two equilibration simulations (NVT and NPT) of $0.5 \mathrm{~ns}$ each. To constrain the bonds involving hydrogen to their equilibrium lengths, we employed P-LINCS ${ }^{53}$ and used a time step of 2 fs. The temperature was kept at $300 \mathrm{~K}$ using the velocity rescaling thermostat of Bussi et al., ${ }^{54}$ and in the case of the NPT equilibration, the pressure was kept at $1 \mathrm{~atm}$ using the semiisotropic Berendsen barostat $(\tau p=5 \mathrm{ps}) .{ }^{55}$ Short-range van der Waals and electrostatic interactions were truncated at $1.2 \mathrm{~nm}$. Long-range electrostatics were computed using the particle mesh Ewald method. ${ }^{56}$ Following equilibration, all five simulation setups were run for $100 \mathrm{~ns}$ at constant pressure using a semi-isotropic Parrinello-Rahman barostat $(\tau p=4$ ps) ${ }^{57}$ The analysis of the simulation data was performed using MDAnalysis (version 0.15$)^{58}$ for Python. Trajectories were visualized using VMD (version 1.9.1). ${ }^{59}$ 
We calculated the probability density of the water oxygen along the surface normal ( $z$-axis). The first peak in the water density profile corresponds to a highly oriented first water layer. This first layer is excluded from Boltzmann inversion to derive the potential of mean force (PMF) (see Supporting Information Figure 12). The hydration force $F$ is the first derivative of the PMF with respect to $z$, and the force gradient is then the second derivative. To compare to experimental data, this force was normalized by an assumed sharp tip with radius of $R=1 \mathrm{~nm}, F / R$.

To capture the orientation of water molecules in the vicinity of the mica surface, we describe the orientation of the dipole moment of water with the order parameter $\langle\cos \theta\rangle$, where $\theta$ is the angle between the dipole vector and the surface normal (see Supporting Information Figure 14). As there was no AFM tip present in the MD simulations, the interaction between the tip and mica was not part of the computationally derived hydration force and force gradient curves. The exponential term of the fit of the experimentally measured force curves (first term of eq 1) was added to the computationally derived oscillatory hydration force curves for comparison.

\section{RESULTS AND DISCUSSION}

Dependence on Specific Ions. Hydration forces were measured using noncontact amplitude modulation AFM. Next, 100-200 approach curves (gray lines in Figure 1a) were

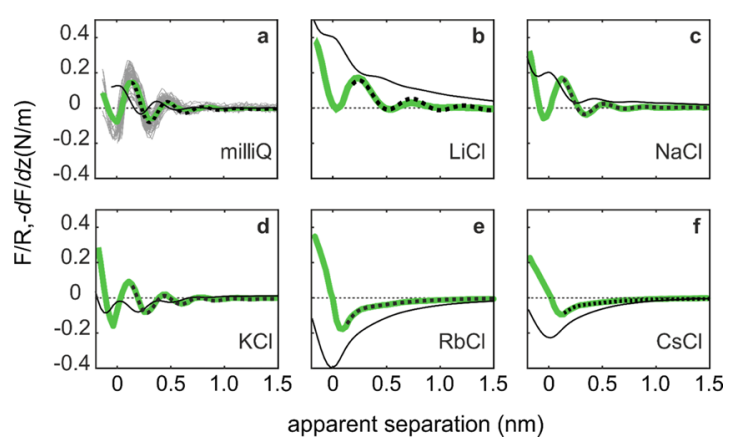

Figure 1. Averaged force gradient $(-\mathrm{d} F / \mathrm{d} z$; thick green lines $)$ and normalized force $(F / R$; thin black lines; nominal tip radius $R=5 \mathrm{~nm})$ versus apparent tip-sample separation measured in (a) pure water (light gray: 180 individual force curves). ( $b-f)$ Various salts at a fixed concentration of $50 \mathrm{mM}$. All data were recorded with the same AFM tip at $\mathrm{pH}$ 6. Dotted lines: empirical fits to eq 1 .

recorded for each fluid composition with the mica substrate and the AFM cantilever $\left(\mathrm{SiO}_{2}\right)$ fully immersed in the electrolyte solution (see Supporting Information for details). The raw data were converted to forces using standard force inversion procedures. ${ }^{45-47,60}$ Subsequent averaging of the individual force gradient curves led to the final force gradient curves (thick green lines in Figure 1) and finally by numerical integration to force curves (thin black lines).

For pure water as well as the strongly hydrated $\mathrm{Li}^{+}, \mathrm{Na}^{+}$, and $\mathrm{K}^{+}$cations, the force gradient curves display a pronounced oscillatory character with up to three local maxima of the force gradient. The observation of oscillatory forces in deionized water (Figure 1a) demonstrates that force oscillations are an intrinsic property of pure confined water and do not require the presence of added salt, in contrast to a widely accepted picture based on SFA measurements. The distance between adjacent maxima (or minima) varies between 0.28 and $0.35 \mathrm{~nm}$ and thus points to a pronounced layered arrangement of the water molecules within the first nanometer from the solid surface. In striking contrast, for $\mathrm{Rb}^{+}$and $\mathrm{Cs}^{+}$solutions, the AFM tip experiences a purely attractive force that increases monotonically from approximately $1 \mathrm{~nm}$ down to $0.1 \mathrm{~nm}$, where a strong repulsion sets in upon tip-sample contact. The oscillatory part of the force is completely suppressed.

Integrating the force gradient data reveals a second ionspecific aspect of the tip-sample interaction force: for the strongly hydrated cations, the oscillatory force is superimposed onto a monotonically decaying repulsive background force. This monotonic force has a slightly larger range and can be detected up to distances of $\approx 1.5 \mathrm{~nm}$. The strength of this force decays from $\mathrm{Li}^{+}$to $\mathrm{Na}^{+}$, almost vanishes for $\mathrm{K}^{+}$, and changes sign to become attractive for $\mathrm{Rb}^{+}$and $\mathrm{Cs}^{+}$. Plotting the logarithm of the absolute value reveals that the force overall decays exponentially with increasing tip-sample distance. The same trends were recorded in several independent experiments with different samples and AFM tips (see Supporting Information Figures 6 and 7).

The qualitative trends can be captured in quantitative numbers by fitting the force gradient data using an empirical function (dashed lines in Figure 1) consisting of a combination of a monotonically decaying exponential function $\phi_{\mathrm{m}}(z)$ and a decaying oscillatory contribution $\phi_{\text {osc }}(z) .^{11,24}$

$$
\phi(z)=\phi_{\mathrm{m}}^{0} \mathrm{e}^{-z / \lambda_{\mathrm{m}}}+\phi_{\mathrm{osc}}^{0} \cos (2 \pi z / d+\varphi) \mathrm{e}^{-z / \lambda_{\mathrm{osc}}}
$$

A monotonic exponential decay is characteristic of hydration forces in many biological (membranes), ${ }^{33,34}$ amorphous silica, $^{31,32}$ and geological (clay swelling) ${ }^{5}$ systems. The additional oscillatory component is typical for rigid atomically smooth surfaces. Fitting to the experimental data reveals that the decay lengths of the oscillatory and the monotonic contribution are $\approx 0.2 \pm 0.08$ and $0.4 \pm 0.1 \mathrm{~nm}$, respectively, and do not display any appreciable dependence on the type of cation. The disappearance of the oscillatory force and the appearance of an attractive monotonic force for $\mathrm{Rb}^{+}$and $\mathrm{Cs}^{+}$ are reflected in the vanishing values of $\phi_{\mathrm{osc}}^{0}$ and the reversal of the sign of $\phi_{\mathrm{m}}^{0}$ (see Supporting Information Figure 1 and Table I for the numerical values of the coefficients).

Concentration Dependence. A possible explanation of the monotonically decaying force is the electrostatic interaction between two effectively charged surfaces interacting across an electrolyte solution, as recently suggested. ${ }^{61}$ The effective surface charge on mica and silica (AFM tip) results from the negative bare surface charge that is partially compensated by specific adsorption of cations. For weakly adsorbing cations, the effective surface charge on both surfaces is expected to be negative, leading to repulsion in solutions of $\mathrm{LiCl}$ and $\mathrm{NaCl}$. On the other hand, more strongly adsorbing cations, such as $\mathrm{Rb}^{+}$and $\mathrm{Cs}^{+}$, could reverse the surface charge of mica or the AFM tip, leading to attraction between the two surfaces with opposite effective charge. ${ }^{62,63}$ If the behavior of the monotonically decaying force was indeed caused by continuum electrostatics in combination with charge regulation, its range would be determined by the Debye screening length $\lambda_{\mathrm{D}}$ and thus vary with the salt concentration. To falsify this assumption, we repeated the measurements for selected salts of variable concentrations (Figure 2). While the details vary slightly, the overall behavior for $\mathrm{Li}^{+}$and $\mathrm{K}^{+}$is essentially the same for all concentrations. All curves display the same oscillatory solvation structure superimposed onto an ion- 


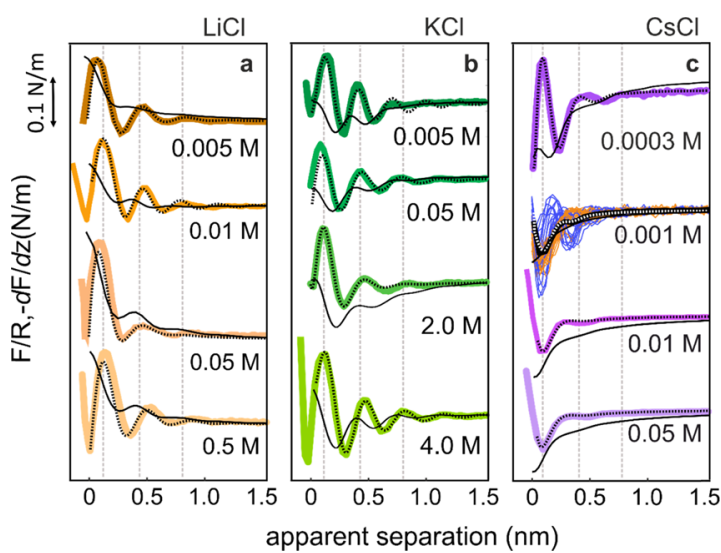

Figure 2. Averaged $(n=50)$ force gradient $(-\mathrm{d} F / \mathrm{d} z$; thick lines $)$ and normalized force $(F / R$; thin black lines) versus apparent tip-sample separation for $\mathrm{LiCl}$ (a) $\mathrm{KCl}$ (b), and $\mathrm{CsCl}$ (c) for several concentrations as indicated. Each salt was measured with a separate AFM tip at $\mathrm{pH}$ 6. Dashed lines: empirical fits to eq 1.

specific but largely concentration-independent monotonic background. In particular, the spacing between local maxima and minima is consistent with the diameter of water up to the highest concentration investigated. In contrast, for $\mathrm{Cs}^{+}$, the force oscillations disappear around a critical concentration of 1 $\mathrm{mM}$. For all concentrations above that threshold, the force becomes purely attractive as already seen in Figure 1.

In the critical concentration range of about $1 \mathrm{mM}, \mathrm{Cs}^{+}$containing solutions display a bistable behavior. Consecutively recorded force curves alternate between the characteristic waterlike response with an oscillatory structure and the purely attractive behavior, which is unique for $\mathrm{Cs}^{+}$and $\mathrm{Rb}^{+}$at high concentrations. Typically, each of the two types of response is observed for several consecutive approaches before the system switches to the other. For this specific concentration, it thus seems that there are two configurations in which the system can get trapped for extended periods of time (4-5 s). Qualitatively, this observation is consistent with the long residence times of $\approx 100 \mathrm{~ms}$ of $\mathrm{Rb}^{+}$ions on mica that were reported in recent AFM measurements ${ }^{64}$ and with the slow ion-exchange rates in $\mathrm{X}$-ray-based $\mathrm{Na}^{+}-\mathrm{Rb}^{+}$ion-exchange reactions. ${ }^{19}$ According to the AFM studies ${ }^{65}$ and recent X-ray measurements, ${ }^{66}$ the mica surface is only partially covered by cations for approximately millimolar concentrations. The steepness of the adsorption isotherm in this concentration range in combination with lateral attractions promotes the formation of patches of adsorbed ions. ${ }^{65}$ Such patches would be expected to display very slow collective dynamics that could explain our observations.

pH Dependence. Early SFA studies of hydration forces reported that the presence and strength of hydration forces require the presence of ions beyond a certain threshold concentration of up to $10 \mathrm{mM} \cdot{ }^{17,18}$ Moreover, the strength of the hydration forces is thought to correlate with the bulk hydration energy of the cations. ${ }^{17}$ Our observations clearly show that for sharp AFM tips, the oscillatory part of the hydration forces can also be observed in the absence of any added salt (Figure 1a). This is consistent with the oscillatory density profiles extracted from X-ray reflectivity measurements ${ }^{19,20}$ as well as MD simulations ${ }^{26,27}$ and supports the idea that the oscillatory part of the hydration forces experienced by an AFM tip indeed originates from the density of the interfacial water. ${ }^{41-43}$ In the absence of added salt, hydronium ions are likely to compensate the intrinsic negative surface charge of mica, as has been suggested by other studies. ${ }^{17,66}$ Interestingly, hydronium ions turn out to have a similarly destructive effect on the oscillatory hydration structure of the interfacial water as the bulky $\mathrm{Rb}^{+}$and $\mathrm{Cs}^{+}$ions. The amplitude of the oscillatory force component decreases with decreasing $\mathrm{pH}$, and a slightly attractive monotonic contribution develops (Figure 3). While

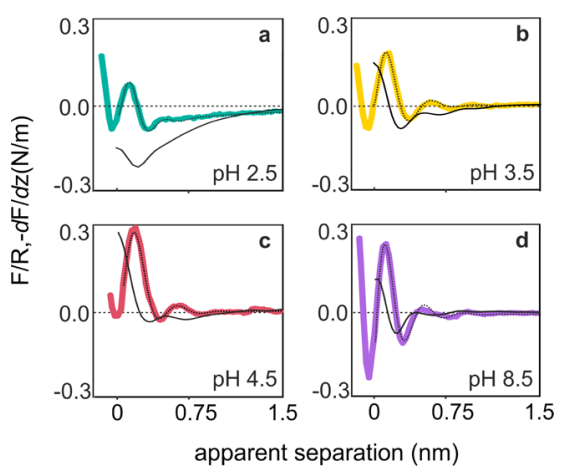

Figure 3. Averaged $(n=50)$ force gradient $(-\mathrm{d} F / \mathrm{d} z$; thick lines $)$ and normalized force $(F / R$; thin black lines) versus apparent tip-sample separation measured at selected bulk $\mathrm{pH}$ values. Dashed line: empirical fit. The $\mathrm{pH}$ was adjusted by adding either $\mathrm{HCl}$ or $\mathrm{NaOH}$.

the suppression is less pronounced than in the case of $\mathrm{Cs}^{+}$ions, the first signs of a reduction can already be detected in $\mathrm{pH} 2.5$, that is, at a similar $\mathrm{H}_{3} \mathrm{O}^{+}$cation concentration as for $\mathrm{Cs}^{+}$.

These observations have several direct implications. First, whenever the forces display an oscillatory component, the spacing between subsequent local force maxima is always close to the diameter of a water molecule, independent of salt concentration and $\mathrm{pH}$. Neither our experiments nor our simulations (see below) display any indication of wider separations due to the potential formation of ion pairs or due to the expulsion of completely hydrated cations. ${ }^{25,35,37} \mathrm{We}$ believe that the smallness of the tip-sample contact in AFM experiments with sharp tips (as compared to SFA and colloidal probe AFM) is essential for observing this intrinsic behavior of the mica-electrolyte interface, which is consistent with the structure of the electric double layers reported in X-ray measurements ${ }^{19,20}$ and MD simulations. ${ }^{26,27}$ Second, because the range of the forces in Figure 2 remains limited to $\approx 1 \mathrm{~nm}$ while the Debye screening length varies by a factor of $\approx 30$, the origin of the monotonic force cannot be attributed to electrostatic screening. Instead, we can rationalize the invariance of the decay length in terms of the phenomenological model by Marčelja and Radić. ${ }^{67}$ According to this mean field model, an exponentially decaying long-range force arises from the polarization of water molecules that are induced at the solid-liquid interface. The decay occurs on a length scale that is governed by the correlation length of the water. The observed invariance of the decay length upon adding salt indeed confirms that the decay is governed by the intrinsic properties of water, largely independent of the added ions. In contrast, the strength of the hydration force is imposed by the degree of order and polarization imposed on the water and thus directly by the microscopic electric fields and hydrogen bonding between the first layer of water and the substrate, including adsorbed ions. Depending on their specific size, adsorption site, and configuration (e.g., inner shell vs outer 
shell), adsorbing ions affect the intrinsic hydration structure of the mica surface in an ion-specific manner. ${ }^{33}$

Third, the salt dependence of the monotonic part of the force cannot be attributed to van der Waals interaction. While definitely contributing to the total force, van der Waals interaction cannot explain the change in sign of the monotonic part of the force upon changing the salt species and concentration. For the present system, the van der Waals force is always attractive with a Hamaker constant (based on bulk properties) that varies between $-8.4 \times 10^{-21} \mathrm{~J}$ (pure water) and $-5.1 \times 10^{-21} \mathrm{~J}(4 \mathrm{M} \mathrm{KCl})$. Hence, we conclude that the ion-specific responses of both the oscillatory and the monotonic part of the tip-sample interaction are dominated by the microscopic hydration structure of the mica-electrolyte interface.

MD Simulations. To analyze the ion-specific structure of interfacial water in more detail, we performed MD simulations for aqueous alkali chloride concentrations $(50 \mathrm{mM})$ as in Figure 1 (see Methods and Supporting Information for details). Cations were found to adsorb to the mica surface to compensate the intrinsic negative surface charge. The preferred adsorption sites were found to shift with increasing ion size from the oxygen triad above an $\mathrm{Al}$ atom for $\mathrm{Li}^{+}$toward the center of the ditrigonal cavity for $\mathrm{Cs}^{+}$(Figure 4 and Supporting Information Figures 10 and 11), in agreement with previous MD simulations. ${ }^{26,27,68}$ Similarly, the corresponding electron density profiles normal to the surface displayed a reasonable agreement with earlier X-ray reflectivity and surface

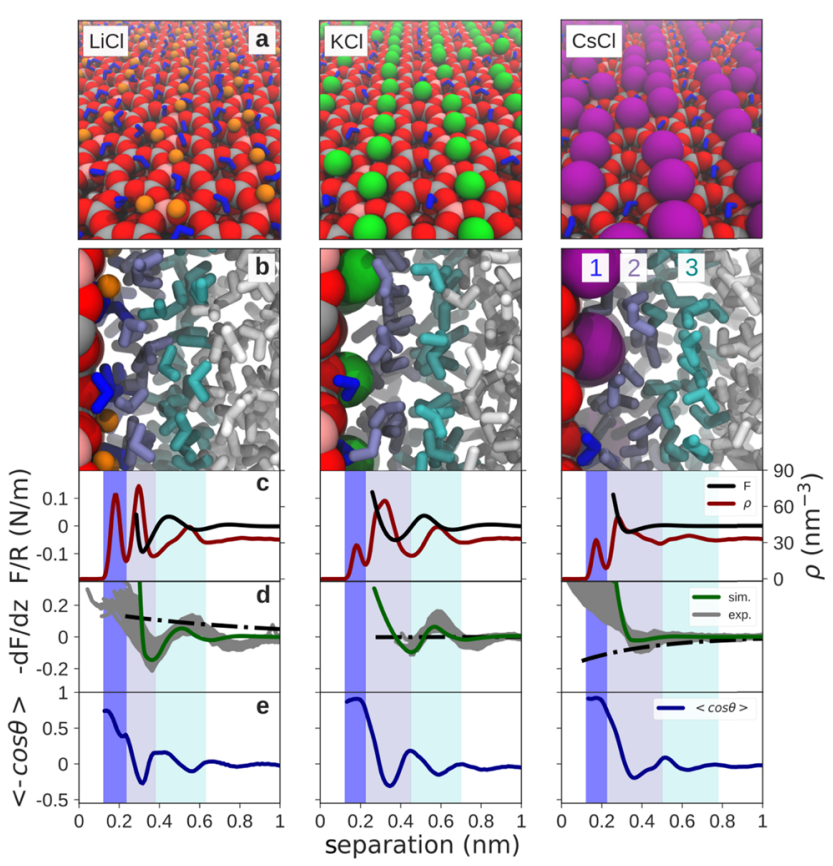

Figure 4. Water structure at the mica-water interface for $\mathrm{LiCl}$ (left), $\mathrm{KCl}$ (middle), and $\mathrm{CsCl}$ (right). (a) Adsorbed ions and oriented water molecules in the first layer. (b) Side view displaying strong water layering in the presence of $\mathrm{Li}^{+}$and $\mathrm{K}^{+}$and the disruption of layering for $\mathrm{Cs}^{+}$. (c) Normalized force (black) and water density profile (red) from MD simulations. (d) Force gradient from MD (green), experimental force gradient curves (gray) after subtraction of monotonic contribution (dashed). (e) Order parameter for orientation of water, defined as $\cos \theta$, where $\theta$ is the angle between the dipole moment of water and surface normal (see Supporting Information Figure 14). diffraction. ${ }^{20,66}$ Cations were found to exchange with the bulk at rates that decrease according to $\mathrm{Na}^{+}>\mathrm{K}^{+}>\mathrm{Cs}^{+}>\mathrm{Li}^{+}$, implying that the forces experienced by the AFM tip on typical measurement time scales (several milliseconds) represent thermally averaged configurations of both interfacial water and adsorbed ions.

The interfacial water structure is characterized by two quantities, the density profile and the water dipole orientation order parameter $\langle\cos \theta\rangle$. In the first water layer in direct contact with the mica surface, water molecules are highly oriented with both hydrogen atoms pointing toward the negatively charged oxygen atoms of the mica crystal (Figure $4 \mathrm{~b})$. The order parameter $\langle\cos \theta\rangle$ indicates that the water dipoles are more strongly oriented for $\mathrm{Cs}^{+}$compared to $\mathrm{Li}^{+}$ (Figure 4e). This results from the fact that small, strongly hydrated cations perturb the substrate-induced orientation of the first water layer more strongly than larger ones. Beyond the first layer, we observe two additional pronounced maxima in the water density for all ions except $\mathrm{Cs}^{+}$. Despite this pronounced positional order, water molecules in the second and third layers display a much weaker preferential orientation (Figure 4e), as also reported elsewhere. ${ }^{69}$ In addition to decreasing orientational order, the maxima in the water density are much less pronounced and broader for $\mathrm{Cs}^{+}$(Figure 4c). Thus, adsorbed $\mathrm{Cs}^{+}$ions disrupt both the orientational and the positional order of interfacial water beyond the first water layer.

To compare the simulation results to the AFM data, we calculated the unit cell-averaged potential of mean force experienced by water molecules as a function of distance from the surface following the procedure proposed by Watkins and Reischl that has been applied successfully for several systems. ${ }^{41-43,61}$ The resulting force and force gradients obtained by differentiating the potential of mean force display oscillations that reflect the oscillations of the water density (Figure 4c,d and Supporting Information Figure 13). The force oscillations thus indeed arise from the layered water structure of the interfacial water. Consistent with the experimental observations, the simulations show that this layered water structure is only weakly disturbed by strongly hydrated cations $\left(\mathrm{Li}^{+}, \mathrm{K}^{+}\right.$, and also by $\mathrm{Na}^{+}$; see Supporting Information Figure 13). In contrast, the bulky $\mathrm{Cs}^{+}$ions, which disturb the water layering, substantially suppress the force oscillations. The local reduction of the water density in the vicinity of the $\mathrm{Cs}^{+}$ions, which is also observed in the bulk, ${ }^{70}$ is crucial for this effect. This leads to shorter residence times of water in the vicinity of $\mathrm{Cs}^{+}$compared to other cations and consequently to weaker hydration forces. ${ }^{27}$

The force gradient based on the numerical calculations agrees almost quantitatively with the oscillatory part of the experimentally measured data (Figure $4 \mathrm{~d}$ ). The simulations reproduce in particular the remarkable robustness of the force oscillations against the presence of small hydrated cations as well as their disappearance in the presence of $\mathrm{Cs}^{+}$. Note that the comparison excludes the region of the first water layer, where the molecules are tightly squeezed in between the adsorbed cations. Most likely, these ions are not displaced by the AFM tip during the experiments. In contrast to the oscillations, the monotonic contribution of the hydration forces is not captured by the simulations. As we will discuss below, detecting this contribution seems to require a "macroscopic" object as a probe, such as the AFM tip. Our observation agrees with X-ray diffraction studies, where it was 
found as well that the interfacial hydration layers are less ordered in the $\mathrm{CsCl}$ solutions compared to solutions containing $\mathrm{K}-, \mathrm{Na}-$, or $\mathrm{LiCl}^{20}$

To interpret these results, we consider the work $\Delta W=\Delta W_{\mathrm{m}}$ $+\Delta W_{\text {osc }}$ performed upon approaching the AFM tip to the sample. For the monotonic contribution of the force, we find values of $\Delta W_{\mathrm{m}}=\iint \phi_{\mathrm{m}}\left(z^{\prime}\right) \mathrm{d} z^{\prime} \mathrm{d} z \approx-2.8, \ldots,+6.4 k T$ for bringing the tip from a distance of 1.5 to $0.2 \mathrm{~nm}$ (red circles in Figure 5a). These values contain contributions from both
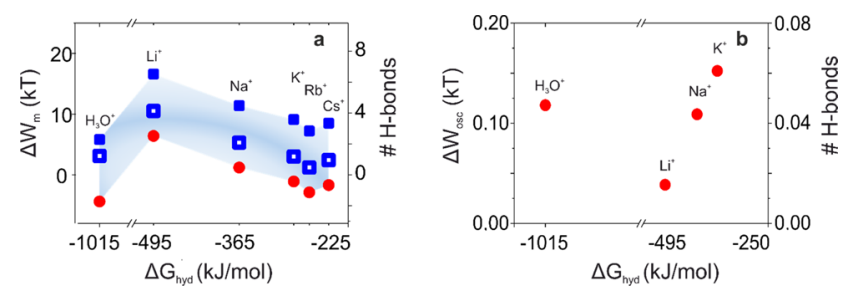

Figure 5. Mechanical work upon approaching the tip and sample in units of $\mathrm{kT} / \mathrm{mol}$ (left axis) and equivalent number of $\mathrm{H}$ bonds (right axis) vs Gibbs free energy of cation hydration in bulk. ${ }^{71}$ Data taken from Figure 1. (a) Red circles are the total work of the monotonic part of the force. Blue filled squares correspond to the total work corrected using van der Waals interaction with macroscopic Hamaker constants; open blue squares are the best estimate for the total hydration work based on the adjusted Hamaker constant. See text for details. (b) Total work of the oscillatory part of the force and equivalent number of $\mathrm{H}$ bonds (right axis).

monotonic hydration forces and direct tip-sample forces due to van der Waals interaction. The latter need to be subtracted to extract the contribution due to hydration. If we use the bulk values of the refractive index and the dielectric constant to calculate the Hamaker constants $A$ for the various electrolytes, we obtain positive values of $\Delta W_{\mathrm{m}}^{\mathrm{hydr}}=\Delta W_{\mathrm{m}}+\frac{A R}{6 D}$ for all salts and concentrations investigated (filled blue squares in Figure $5 a$ and Supporting Information Figures 2-5).

However, estimating the direct tip-sample interaction based on bulk fluid properties provides an overestimation of van der Waals interactions because the dielectric constant decreases close to the substrate ${ }^{72}$ due to hindered rotation of the water molecules and the refractive index increases (in particular for the heavier cations) due to the enhanced local ion concentration. Correcting for the reduced Hamaker constant (see Supporting Information for details) results in a best estimate for $\Delta W_{\mathrm{m}}^{\mathrm{hydr}}$, which is positive for all salts, implying repulsive hydration forces (open blue squares in Figure 5a and Supporting Information Figures 2-5). With the exception of $\mathrm{H}_{3} \mathrm{O}^{+}$, the strength of the hydration force is found to decrease with increasing ion radius, largely following the order of the bulk hydration energy (Figure 5a).

It is also important to appreciate the smallness of the absolute value of $\Delta W_{\mathrm{m}}^{\mathrm{hydr}}$ of only a few $\mathrm{kT}$. Using the tip geometry and the bulk density of water $\left(\rho_{\mathrm{H}_{2} \mathrm{O}}=33 \mathrm{~nm}^{-1}\right)$, we estimate that this amount of work is distributed over $\approx 1000$ water molecules that are transferred from the interfacial region between the tip and sample to the ambient bulk. In the final state, these molecules are engaged in $\approx 2000 \mathrm{H}$ bonds (avoiding double counting). Given the strength of a $\mathrm{H}$ bond of $\approx \mathrm{kT}$ (without separating the molecules), ${ }^{73}$ we conclude that the changes in the water structure correspond to the equivalent of breaking only a few $\mathrm{H}$ bonds. Taking the thickness of the interfacial water layer to be $1 \mathrm{~nm}, 1$ in $1000 \mathrm{H}$ bonds corresponds to an excess interfacial energy of $\approx 0.4 \mathrm{~mJ} / \mathrm{m}^{2}$. Compared to the bulk cohesive energy and the total micaelectrolyte interfacial energy, the average perturbation per water molecule due to hydration effects is thus rather weak. ${ }^{74,75}$ Presumably, this also explains why the monotonic contribution of the force is not resolved in our (and other) MD simulations based on the potential of the mean force approach for a single water molecule. These forces are only resolved in measurements and simulations that explicitly include a macroscopic object such as an AFM tip, which integrates the forces due to a large number of water molecules. A similar argument has been discussed in the context of hydration pressures in biological systems. ${ }^{16}$

Performing the same analysis for the oscillatory part of the hydration forces yields a somewhat different picture. Integration over several oscillations leads to minor values for $\Delta W_{\text {osc }}=\iint \phi_{\text {osc }}\left(z^{\prime}\right) \mathrm{d} z^{\prime} \mathrm{d} z$ because of cancellation effects between regions with attractive and repulsive forces. Yet, even upon displacing the tip, say, from the first minimum in the force gradient at a separation of $\approx 0.3 \mathrm{~nm}$ to the first maximum at $\approx 0.15 \mathrm{~nm}$, also $\Delta W_{\text {osc }}$ amounts only to a fraction of $\mathrm{kT}$ (Figure $5 \mathrm{~b}$ ), that is, substantially less than the binding energy of a single $\mathrm{H}$ bond. Therefore, we conclude that the work involved in the tip displacement must be absorbed by a slight distortion of the dynamically reorganizing $\mathrm{H}$-bonding network among the water molecules involved in the process. Their number, however, is more difficult to estimate than above because it depends strongly on the details of the tip geometry. MD simulations of atomically resolved AFM images with explicit tips suggest that only very few water molecules are involved; ${ }^{42}$ geometric estimates provide an upper limit of a few tens of molecules.

The dependence on the ionic species (and thus the bulk hydration energy) of $\Delta W_{\text {osc }}$ is very different from the monotonic hydration force. Given the locality of the interactions, it is in fact quite plausible that the details of the local binding environment provided by the surface and the adsorbed cations control the ion specificity of $\Delta W_{\text {osc }}$ rather than the bulk hydration energy. This is consistent with the numerically calculated water density profiles.

\section{CONCLUSIONS}

In summary, our work shows that the hydration forces at smooth mica-electrolyte interfaces are composed of two contributions, an oscillatory one that is determined by the direct interactions among solid, ions, and water on the scale of one to two molecular layers and a monotonically decaying one that ranges up to three or four water layers. While the overall strength of the hydration interactions amounts to the equivalent of breaking only a few $\mathrm{H}$ bonds, the dependence on the cation species decreases by up to a factor of 4 between strongly hydrated $\mathrm{Li}^{+}$ions and weakly hydrated $\mathrm{Rb}^{+}$and $\mathrm{Cs}^{+}$ ions. The latter two cations completely disrupt the oscillatory force profiles that are characteristic for the mica-water interface, whereas the profile remains stable in the presence of the more strongly hydrated alkali ions. These findings shed light on the relative importance of different aspects of solvation and hydration for chemical reactions involving small molecules (e.g., in catalytic processes ${ }^{10}$ ) as compared to contact formation, adhesion, friction, etc. on the colloidal scale. 


\section{ASSOCIATED CONTENT}

\section{S Supporting Information}

The Supporting Information is available free of charge on the ACS Publications website at DOI: 10.1021/acs.langmuir.9b00520.

Details of fitting procedure, van der Waals interaction and Hamaker constant, MD simulation in pure water, atomistic model for mica, preferred binding sites, potential of mean force, results from MD simulations for pure water and $\mathrm{NaCl}$, and SEM images of a used AFM probe (PDF)

\section{AUTHOR INFORMATION}

\section{Corresponding Author}

*E-mail: f.mugele@utwente.nl.

\section{ORCID $\odot$}

Igor Siretanu: 0000-0002-5741-9561

\section{Notes}

The authors declare no competing financial interest.

\section{ACKNOWLEDGMENTS}

This work is part of the research program Rock-on-a-Chip with project number i40, which is partly financed by the Netherlands Organisation for Scientific Research (NWO) and through the Exploratory Research (ExploRe) program of BP plc. BP Exploration Operating Company Limited is thanked for permission to publish this paper. K.K.G. and N.S. acknowledge financial support from the DFG (Emmy Noether program). We thank M. Haagh for his help writing codes used for the analysis of the AFM data.

\section{REFERENCES}

(1) Israelachvili, J.; Wennerström, H. Role of hydration and water structure in biological and colloidal interactions. Nature 1996, 379, 219.

(2) Lis, L. J.; McAlister, M.; Fuller, N.; Rand, R.; Parsegian, V. Interactions between neutral phospholipid bilayer membranes. Biophys. J. 1982, 37, 657.

(3) Rau, D. C.; Lee, B.; Parsegian, V. Measurement of the repulsive force between polyelectrolyte molecules in ionic solution: hydration forces between parallel DNA double helices. Proc. Natl. Acad. Sci. U.S.A. 1984, 81, 2621-2625.

(4) Valle-Delgado, J.; Molina-Bolívar, J.; Galisteo-González, F.; Gálvez-Ruiz, M. Evidence of hydration forces between proteins. Curr. Opin. Colloid Interface Sci. 2011, 16, 572-578.

(5) Viani, B. E.; Low, P. F.; Roth, C. B. Direct measurement of the relation between interlayer force and interlayer distance in the swelling of montmorillonite. J. Colloid Interface Sci. 1983, 96, 229244.

(6) Mugele, F.; Bera, B.; Cavalli, A.; Siretanu, I.; Maestro, A.; Duits, M.; Cohen-Stuart, M.; Van Den Ende, D.; Stocker, I.; Collins, I. Ion adsorption-induced wetting transition in oil-water-mineral systems. Sci. Rep. 2015, 5, No. 10519.

(7) Gong, X.; Wang, B.; Kozbial, A.; Li, L. From Molecular Arrangement to Macroscopic Wetting of Ionic Liquids on the Mica Surface: Effect of Humidity. Langmuir 2018, 34, 12167-12173.

(8) Israelachvili, J. N. Adhesion forces between surfaces in liquids and condensable vapours. Surf. Sci. Rep. 1992, 14, 109-159.

(9) Ma, L.; Gaisinskaya-Kipnis, A.; Kampf, N.; Klein, J. Origins of hydration lubrication. Nat. Commun. 2015, 6, No. 6060.

(10) Ledezma-Yanez, I.; Wallace, W. D. Z.; Sebastián-Pascual, P.; Climent, V.; Feliu, J. M.; Koper, M. T. Interfacial water reorganization as a $\mathrm{pH}$-dependent descriptor of the hydrogen evolution rate on platinum electrodes. Nat. Energy 2017, 2, No. 17031.
(11) Israelachvili, J. N. Intermolecular and Surface Forces; Academic press, 2011.

(12) Derjaguin, B.; Landau, L. The theory of stability of highly charged lyophobic sols and coalescence of highly charged particles in electrolyte solutions. Acta Physicochim. URSS 1941, 14, 58.

(13) Verwey, E. J. W.; Overbeek, J. T. G.; Overbeek, J. T. G. Theory of the Stability of Lyophobic Colloids; Courier Corporation, 1999.

(14) Zhao, C.; Ebeling, D.; Siretanu, I.; van den Ende, D.; Mugele, F. Extracting local surface charges and charge regulation behavior from atomic force microscopy measurements at heterogeneous solidelectrolyte interfaces. Nanoscale 2015, 7, 16298-16311.

(15) Ruiz-Cabello, F. J. M.; Trefalt, G.; Maroni, P.; Borkovec, M. Electric double-layer potentials and surface regulation properties measured by colloidal-probe atomic force microscopy. Phys. Rev. E 2014, 90, No. 012301.

(16) Parsegian, V.; Zemb, T. Hydration forces: Observations, explanations, expectations, questions. Curr. Opin. Colloid Interface Sci. 2011, 16, 618-624.

(17) Pashley, R. DLVO and hydration forces between mica surfaces in $\mathrm{Li}+, \mathrm{Na}+, \mathrm{K}+$, and $\mathrm{Cs}+$ electrolyte solutions: A correlation of double-layer and hydration forces with surface cation exchange properties. J. Colloid Interface Sci. 1981, 83, 531-546.

(18) Israelachvili, J. N.; Pashley, R. M. Molecular layering of water at surfaces and origin of repulsive hydration forces. Nature 1983, 306, 249.

(19) Lee, S. S.; Fenter, P.; Nagy, K. L.; Sturchio, N. C. Real-time observation of cation exchange kinetics and dynamics at the muscovite-water interface. Nat. Commun. 2017, 8, No. 15826.

(20) Lee, S. S.; Fenter, P.; Nagy, K. L.; Sturchio, N. C. Monovalent ion adsorption at the muscovite (001)-solution interface: Relationships among ion coverage and speciation, interfacial water structure, and substrate relaxation. Langmuir 2012, 28, 8637-8650.

(21) Pintea, S.; de Poel, W.; de Jong, A. E.; Vonk, V.; van der Asdonk, P.; Drnec, J.; Balmes, O.; Isern, H.; Dufrane, T.; Felici, R.; Vlieg, E. Solid-liquid interface structure of muscovite mica in $\mathrm{CsCl}$ and $\mathrm{RbBr}$ solutions. Langmuir 2016, 32, 12955-12965.

(22) Miranda, P.; Xu, L.; Shen, Y.; Salmeron, M. Icelike water monolayer adsorbed on mica at room temperature. Phys. Rev. Lett. 1998, 81, No. 5876.

(23) Fukuma, T.; Ueda, Y.; Yoshioka, S.; Asakawa, H. Atomic-scale distribution of water molecules at the mica-water interface visualized by three-dimensional scanning force microscopy. Phys. Rev. Lett. 2010, 104, No. 016101.

(24) Kilpatrick, J. I.; Loh, S.-H.; Jarvis, S. P. Directly probing the effects of ions on hydration forces at interfaces. J. Am. Chem. Soc. 2013, 135, 2628-2634.

(25) Martin-Jimenez, D.; Chacon, E.; Tarazona, P.; Garcia, R. Atomically resolved three-dimensional structures of electrolyte aqueous solutions near a solid surface. Nat. Commun. 2016, 7, No. 12164

(26) Bourg, I. C.; Lee, S. S.; Fenter, P.; Tournassat, C. Stern layer structure and energetics at mica-water interfaces. J. Phys. Chem. C 2017, 121, 9402-9412.

(27) Sakuma, H.; Kawamura, K. Structure and dynamics of water on $\mathrm{Li}+-, \mathrm{Na}+-, \mathrm{K}+-, \mathrm{Cs}+-, \mathrm{H} 3 \mathrm{O}+$-exchanged muscovite surfaces: A molecular dynamics study. Geochim. Cosmochim. Acta 2011, 75, 6381.

(28) Park, S.-H.; Sposito, G. Structure of water adsorbed on a mica surface. Phys. Rev. Lett. 2002, 89, No. 085501.

(29) Gaigeot, M.-P.; Sprik, M.; Sulpizi, M. Oxide/water interfaces: how the surface chemistry modifies interfacial water properties. $J$. Phys.: Condens. Matter 2012, 24, No. 124106.

(30) Goldberg, R.; Chai, L.; Perkin, S.; Kampf, N.; Klein, J. Breakdown of hydration repulsion between charged surfaces in aqueous Cst solutions. Phys. Chem. Chem. Phys. 2008, 10, 49394945.

(31) Ducker, W. A.; Senden, T. J.; Pashley, R. M. Measurement of forces in liquids using a force microscope. Langmuir 1992, 8, 18311836. 
(32) Vigil, G.; Xu, Z.; Steinberg, S.; Israelachvili, J. Interactions of silica surfaces. J. Colloid Interface Sci. 1994, 165, 367-385.

(33) Kanduč, M.; Schlaich, A.; Schneck, E.; Netz, R. R. Hydration repulsion between membranes and polar surfaces: Simulation approaches versus continuum theories. Adv. Colloid Interface Sci. 2014, 208, 142-152.

(34) Schneck, E.; Sedlmeier, F.; Netz, R. R. Hydration repulsion between biomembranes results from an interplay of dehydration and depolarization. Proc. Natl. Acad. Sci. U.S.A. 2012, 109, 14405-14409.

(35) Espinosa-Marzal, R. M.; Drobek, T.; Balmer, T.; Heuberger, M. P. Hydrated-ion ordering in electrical double layers. Phys. Chem. Chem. Phys. 2012, 14, 6085-6093.

(36) Zachariah, Z.; Espinosa-Marzal, R. M.; Heuberger, M. P. Ion specific hydration in nano-confined electrical double layers. J. Colloid Interface Sci. 2017, 506, 263-270.

(37) Baimpos, T.; Shrestha, B. R.; Raman, S.; Valtiner, M. Effect of Interfacial Ion Structuring on Range and Magnitude of Electric Double Layer, Hydration, and Adhesive Interactions between Mica Surfaces in $0.05-3 \mathrm{M} \mathrm{Li}+$ and Cs+ Electrolyte Solutions. Langmuir 2014, 30, 4322-4332.

(38) Flores, S. C.; Kherb, J.; Cremer, P. S. Direct and reverse Hofmeister effects on interfacial water structure. J. Phys. Chem. C 2012, 116, 14408-14413.

(39) DeWalt-Kerian, E. L.; Kim, S.; Azam, M. S.; Zeng, H.; Liu, Q.; Gibbs, J. M. pH-dependent inversion of Hofmeister trends in the water structure of the electrical double layer. J. Phys. Chem. Lett. 2017, $8,2855-2861$.

(40) Miyazawa, K.; Kobayashi, N.; Watkins, M.; Shluger, A. L.; Amano, K.-i.; Fukuma, T. A relationship between three-dimensional surface hydration structures and force distribution measured by atomic force microscopy. Nanoscale 2016, 8, 7334-7342.

(41) Reischl, B.; Watkins, M.; Foster, A. S. Free energy approaches for modeling atomic force microscopy in liquids. J. Chem. Theory Comput. 2013, 9, 600-608.

(42) Fukuma, T.; Reischl, B.; Kobayashi, N.; Spijker, P.; Canova, F. F.; Miyazawa, K.; Foster, A. S. Mechanism of atomic force microscopy imaging of three-dimensional hydration structures at a solid-liquid interface. Phys. Rev. B 2015, 92, No. 155412.

(43) Watkins, M.; Reischl, B. A simple approximation for forces exerted on an AFM tip in liquid. J. Chem. Phys. 2013, 138, No. 154703.

(44) Söngen, H.; Marutschke, C.; Spijker, P.; Holmgren, E.; Hermes, I.; Bechstein, R.; Klassen, S.; Tracey, J.; Foster, A. S.; Kühnle, A. Chemical Identification at the Solid-Liquid Interface. Langmuir 2017, 33, 125-129.

(45) De Beer, S.; Van den Ende, D.; Mugele, F. Dissipation and oscillatory solvation forces in confined liquids studied by smallamplitude atomic force spectroscopy. Nanotechnology 2010, 21, No. 325703.

(46) Liu, F.; Zhao, C.; Mugele, F.; van den Ende, D. Amplitude modulation atomic force microscopy, is acoustic driving in liquid quantitatively reliable? Nanotechnology 2015, 26, No. 385703.

(47) Butt, H. -J.; Cappella, B.; Kappl, M. Force measurements with the atomic force microscope: Technique, interpretation and applications. Surf. Sci. Rep. 2005, 59, 1-152.

(48) Richardson, S. M.; Richardson, J. W. Crystal structure of a pink muscoyite from Archer's Post, Kenya: implications for reverse pleochroism in dioctahedral micas. Am. Mineralogist 1982, 67, 69-75.

(49) Cygan, R. T.; Liang, J.-J.; Kalinichev, A. G. Molecular models of hydroxide, oxyhydroxide, and clay phases and the development of a general force field. J. Phys. Chem. B 2004, 108, 1255-1266.

(50) Jorgensen, W. L.; Chandrasekhar, J.; Madura, J. D.; Impey, R. W.; Klein, M. L. Comparison of simple potential functions for simulating liquid water. J. Chem. Phys. 1983, 79, 926-935.

(51) Mamatkulov, S.; Schwierz, N. Force fields for monovalent and divalent metal cations in TIP3P water based on thermodynamic and kinetic properties. J. Chem. Phys. 2018, 148, No. 074504.

(52) Hess, B.; Kutzner, C.; Van Der Spoel, D.; Lindahl, E. GROMACS 4: algorithms for highly efficient, load-balanced, and scalable molecular simulation. J. Chem. Theory Comput. 2008, 4, 435447.

(53) Hess, B.; Bekker, H.; Berendsen, H. J.; Fraaije, J. G. LINCS: a linear constraint solver for molecular simulations. J. Comput. Chem. 1997, 18, 1463-1472.

(54) Bussi, G.; Donadio, D.; Parrinello, M. Canonical sampling through velocity rescaling. J. Chem. Phys. 2007, 126, No. 014101.

(55) Berendsen, H. J.; Postma, J.; van Gunsteren, W. F.; DiNola, A.; Haak, J. Molecular dynamics with coupling to an external bath. J. Chem. Phys. 1984, 81, 3684-3690.

(56) Darden, T.; York, D.; Pedersen, L. Particle mesh Ewald: An N. $\log (\mathrm{N})$ method for Ewald sums in large systems. J. Chem. Phys. 1993, 98, 10089-10092.

(57) Parrinello, M.; Rahman, A. Polymorphic transitions in single crystals: A new molecular dynamics method. J. Appl. Phys. 1981, 52, $7182-7190$.

(58) Michaud-Agrawal, N.; Denning, E. J.; Woolf, T. B.; Beckstein, O. MDAnalysis: a toolkit for the analysis of molecular dynamics simulations. J. Comput. Chem. 2011, 32, 2319-2327.

(59) Humphrey, W.; Dalke, A.; Schulten, K. VMD: visual molecular dynamics. J. Mol. Graphics 1996, 14, 33-38.

(60) Klaassen, A.; Liu, F.; Van den Ende, D.; Mugele, F.; Siretanu, I. Impact of surface defects on the surface charge of gibbsite nanoparticles. Nanoscale 2017, 9, 4721-4729.

(61) Umeda, K.; Zivanovic, L.; Kobayashi, K.; Ritala, J.; Kominami, H.; Spijker, P.; Foster, A. S.; Yamada, H. Atomic-resolution threedimensional hydration structures on a heterogeneously charged surface. Nat. Commun. 2017, 8, No. 2111.

(62) Schwierz, N.; Horinek, D.; Sivan, U.; Netz, R. R. Reversed Hofmeister series-the rule rather than the exception. Curr. Opin. Colloid Interface Sci. 2016, 23, 10-18.

(63) Lima, E. R.; Boström, M.; Schwierz, N.; Sernelius, B. E.; Tavares, F. W. Attractive double-layer forces between neutral hydrophobic and neutral hydrophilic surfaces. Phys. Rev. E 2011, 84, No. 061903.

(64) Ricci, M.; Trewby, W.; Cafolla, C.; Voïtchovsky, K. Direct observation of the dynamics of single metal ions at the interface with solids in aqueous solutions. Sci. Rep. 2017, 7, No. 43234.

(65) Ricci, M.; Spijker, P.; Voïtchovsky, K. Water-induced correlation between single ions imaged at the solid-liquid interface. Nat. Commun. 2014, 5, No. 4400.

(66) Brugman, S. J. T.; Townsend, E. R.; Smets, M. M. H.; Accordini, P.; Vlieg, E. Concentration-Dependent Adsorption of CsI at the Muscovite-Electrolyte Interface. Langmuir 2018, 34, 38213826.

(67) Marčelja, S.; Radić, N. Repulsion of interfaces due to boundary water. Chem. Phys. Lett. 1976, 42, 129-130.

(68) Kobayashi, K.; Liang, Y.; Murata, S.; Matsuoka, T.; Takahashi, S.; Nishi, N.; Sakka, T. Ion distribution and hydration structure in the stern layer on muscovite surface. Langmuir 2017, 33, 3892-3899.

(69) Adapa, S.; Swamy, D. R.; Kancharla, S.; Pradhan, S.; Malani, A. Role of Mono- and Divalent Surface Cations on the Structure and Adsorption Behavior of Water on Mica Surface. Langmuir 2018, 34, 14472-14488.

(70) Caralampio, D. Z.; Martínez, J. M.; Pappalardo, R. R.; Marcos, E. S. The hydration structure of the heavy-alkalines $\mathrm{Rb}+$ and Cs+ through molecular dynamics and X-ray absorption spectroscopy: surface clusters and eccentricity. Phys. Chem. Chem. Phys. 2017, 19, 28993-29004.

(71) Marcus, Y. Thermodynamics of solvation of ions. Part 5.Gibbs free energy of hydration at 298.15 K. J. Chem. Soc., Faraday Trans. 1991, 87, 2995-2999.

(72) Schlaich, A.; Knapp, E. W.; Netz, R. R. Water dielectric effects in planar confinement. Phys. Rev. Lett. 2016, 117, No. 048001.

(73) Smith, J. D.; Cappa, C. D.; Wilson, K. R.; Messer, B. M.; Cohen, R. C.; Saykally, R. J. Energetics of hydrogen bond network rearrangements in liquid water. Science 2004, 306, 851-853. 
(74) Cleveland, J.; Schäffer, T.; Hansma, P. Probing oscillatory hydration potentials using thermal-mechanical noise in an atomicforce microscope. Phys. Rev. B 1995, 52, No. R8692.

(75) Ho, R.; Yuan, J.-Y.; Shao, Z. Hydration force in the atomic force microscope: a computational study. Biophys. J. 1998, 75, 10761083. 